Technical note

\title{
A G-protein-coupled chemokine receptor: A putative insertion site for a multi-pathogen recombinant capripoxvirus vaccine strategy
}

\author{
Catherine Cêtre-Sossah a,b,*, Simon Dickmu ${ }^{\text {c }}$, Olivier Kwiatek ${ }^{\text {b,d }}$, Emmanuel Albina ${ }^{\text {b,e }}$ \\ a CIRAD UMR ASTRE, F-97490 Sainte Clotilde, France \\ b INRA, UMR1309 CMAEE, F-34398 Montpellier, France \\ c Laboratoire National Vétérinaire, PO Box 503, Garoua, Cameroon \\ d CIRAD UMR ASTRE, F-34398 Montpellier, France \\ e CIRAD UMR ASTRE, F-97170 Petit-Bourg, France
}

\section{A R T I C L E I N F O}

\section{Article history:}

Received 6 February 2017

Received in revised form 19 May 2017

Accepted 19 May 2017

Available online 31 May 2017

\section{Keywords:}

New insertion site

Capripoxvirus

Vaccine

Goats

\begin{abstract}
A B S T R A C T
Capripoxviruses (CaPVs) have been shown to be ideal viral vectors for the development of recombinant multivalent vaccines to enable delivery of immunogenic genes from ruminant pathogens. So far, the viral thymidine kinase (TK) gene is the only gene used to generate recombinants. A putative non-essential gene encoding a G-protein-coupled chemokine receptor subfamily homologue (GPCR) was targeted as an additional insertion site. Peste des petits ruminants (PPR) was chosen as a disease model. A new recombinant CaPV expressing the viral attachment hemagglutinin (H) of the PPR virus (PPRV) in the GPCR insertion site (rKS1-HPPR-GPCR) was generated in the backbone North African isolate KS1 strain of lumpy skin disease virus (LSDV). Comparison with the recombinant CaPV expressing the H of PPRV in the TK gene (rKS1-HPPR-TK) shown to induce protection against both PPR and LSD in both sheep and goats was assessed. The suitability of the GPCR gene to be a putative additional insertion site in the CaPV genome is evaluated and discussed.
\end{abstract}

(c) 2017 Elsevier B.V. All rights reserved.

\section{Introduction}

Sheep pox virus (SPPV) and goat pox virus (GTPV), along with lumpy skin disease virus (LSDV), a pathogen specific to cattle, are members of the viral genus Capripoxvirus which cause primary viral diseases that have a significant impact on the livestock industry.

Capripoxviruses (CaPVs) have been shown to be ideal viral vectors for the development of recombinant multivalent vaccines to enable delivery of immunogenic genes from ruminant pathogens which share the same geographical distribution (Africa, the Middle East and Asia) (Diallo et al. 2002). They are thermo-resistant and their genome enables insertion of a large recombinant DNA sequence. The live attenuated strain of LSDV, Kenya Sheep 1 (KS1) (Kitching et al. 1987) and the South African vaccine strain of LSDV have been used as vaccine vectors against livestock diseases such as rinderpest, peste des petits ruminants (PPR), bluetongue and Rift Valley fever (Boshra et al. 2013; Diallo et al. 2002; Diallo, 2003; Wallace and Viljoen, 2005; Perrin et al. 2007; Soi et al. 2010). The strategy to generate pox virus recombinants is based on the homologous recombination between a specific gene of the pox virus genome and the flanking regions of the same specific gene encompassing both the transgene to be expressed and a dominant selectable marker. The viral thymidine kinase

\footnotetext{
* Corresponding author at: CIRAD UMR ASTRE/INRA UMR1309, 2, Rue Maxime Rivière, F-97490 Sainte Clotilde, France.

E-mail address: catherine.cetre-sossah@cirad.fr (C. Cêtre-Sossah).
}

(TK) gene, which was found to be non-essential for normal growth, was first used to generate recombinants in the Vaccinia virus and later in the CaPVs (Diallo et al. 2002). A challenge facing the development of a successful vector is generating several insertion sites for multi-gene expression that do not impair replication of the virus. A putative non-essential gene encoding a G-protein-coupled chemokine receptor subfamily homologue (GPCR) was targeted for homologous recombination using the backbone North African isolate KS1 strain of LSDV (Cao et al. 1995; Le Goff et al. 2009).

Peste des petits ruminants (PPR) is one of the most contagious diseases affecting small ruminants. A new recombinant CaPV expressing the viral attachment hemagglutinin $(\mathrm{H})$ of the PPR virus (PPRV) in the GPCR insertion site (rKS1-HPPR-GPCR) was generated. Comparison with the recombinant CaPV expressing the $\mathrm{H}$ of PPRV in the TK gene (rKS1-HPPR-TK) shown to induce protection against both PPR and LSD in both sheep and goats (Caufour et al. 2014) was assessed. The aim of this study was to evaluate the suitability of the GPCR gene as a putative additional insertion site in the CaPV genome.

\section{Materials and methods}

\subsection{Cells and viruses}

The ovine lamb testis cell line OA3.Ts (ATCC CRL-6546) and Vero cells (ATCC CCL681) were used to propagate CaPV and PPRV respectively. All 
the viruses were obtained from the virus bank of CIRAD, Montpellier: attenuated CaPV vaccine strain KS1 (Kenya KS-GP 0240 strain (Kitching et al. 1987), attenuated PPRV vaccine strain Nigeria 75/1 (Diallo, 2003), CaPV (KS1 based) containing the PPRV-H gene into the TK insertion site (Diallo et al. 2002), CaPV (KS1 based) containing the PPRV-H gene into the GPCR insertion site (this study) and the virulent field strains of GTPV Yemen and PPRV India 94/1.

\subsection{Generation of the recombinant capripoxvirus rKS1-HPPR-GPCR}

The GPCR flanking regions GPCR1 and GPCR2 of the KSGP 0240 (KS1) strain were inserted by enzymatic digestion at the sites KpnI/Sfil and BstXI respectively in the previously described plasmid pFPJ2 (Berhe et al. 2003). The Escherichia coli xanthine-guanine phosphoribosyltransferase gene (Ecogpt) was used as a dominant selectable marker to isolate the recombinant. The Ecogpt-p7.5-p7.5 PPR F gene cassette was replaced by the Ecogpt-PS-PS-PPR H gene cassette thereby allowing the H-PPR gene and the Ecogpt to be placed under the control of the vaccinia virus early/late synthetic promoter (a generous gift from Dr. Drillien, University of Strasbourg, France). To check the insertion of the inserted gene in the cassette, specific PCR based on the GPCR flanking regions and the inserted gene H-PPRV have been performed. Final constructions were checked and compared in transient expression assays consisting of the transfection of lamb testis cells (OA3Ts) and infection with the capripoxvirus vaccine strain KS-1. Validated constructions were further used to generate recombinant KS1 capripoxviruses by infection-transfection of OA3Ts as described previously (Perrin et al. 2007). The purity of the recombinant virus was confirmed by the absence of detectable parental virus using a PCR performed directly on DNA extracted from the viral supernatant targeting the GPCR gene.

\subsection{Immunization and experimental challenge}

An animal experiment was carried out on four groups of three Guinea Dwarf goats each, injected subcutaneously with a dose of
$10^{3} \mathrm{TCID}_{50}$ /animal (50\% Tissue culture infective dose) with either rKS1-HPPR-TK, rKS1-HPPR-GPCR, the attenuated CaPV KS1 vaccine (PPRV challenge positive control group) or Vero cell supernatant (mock, CaPV challenge positive control group). To limit the number of animals in the experiment, only two animals were included in the mock group. The virulent Yemen GPV and the India 94 PPRV strains were sequentially used as challenge in all groups at day 21 and day 35 post immunization respectively at a dose of $10^{3} \mathrm{TCID}_{50}$ /animal (Fig. 1).

All experimental procedures were in accordance with ethical standards. The study was approved by the Ethics Committee for Animal Experimentation of CIRAD, Montpellier, France (permit F 34-172-15). Serum samples collected prior to immunization and weekly over an 8week period post immunization were analyzed for H-PPRV antibodies by ELISA (Libeau et al., 1995) and confirmed by VNT (OIE 2012; Rossiter et al. 1985).

All the animals were observed daily for the appearance of clinical signs which were used collectively to give a final severity score for each animal: overall behavior (good, 1 pt; apathy, 1 pt; prostration, 2 pts; recumbence, $3 \mathrm{pts}$ ), clinical signs (rectal temperature, $39{ }^{\circ} \mathrm{C}$ or above, $1 \mathrm{pt}$; cutaneous lesions at the site of injection, $1 \mathrm{pt}$; other extensive cutaneous lesions, 2 pts; respiratory problems [mild nasal discharge, 1 pt; severe nasal discharge, 2 pts; mild ocular discharge, 1 pt; severe ocular discharge, 2 pts, cough, $1 \mathrm{pt}$ ); digestive problems [mild stomatitis (with oral lesions), $1 \mathrm{pt}$; necrotizing stomatitis (with oral lesions), 3 pts; diarrhea, 3 pts]; death, 20 pts. The score given for one animal in Fig. 2 corresponds to the total number of points accumulated during the experiment (from the day of the challenge until the day of euthanasia) divided by the number of days the animal presented clinical signs.

\section{Results and discussion}

The virulent Yemen GPV and the India 94 PPRV strains sequentially used as challenge in all four groups of goats produced the expected severe clinical signs in the respective control group. The two animals in

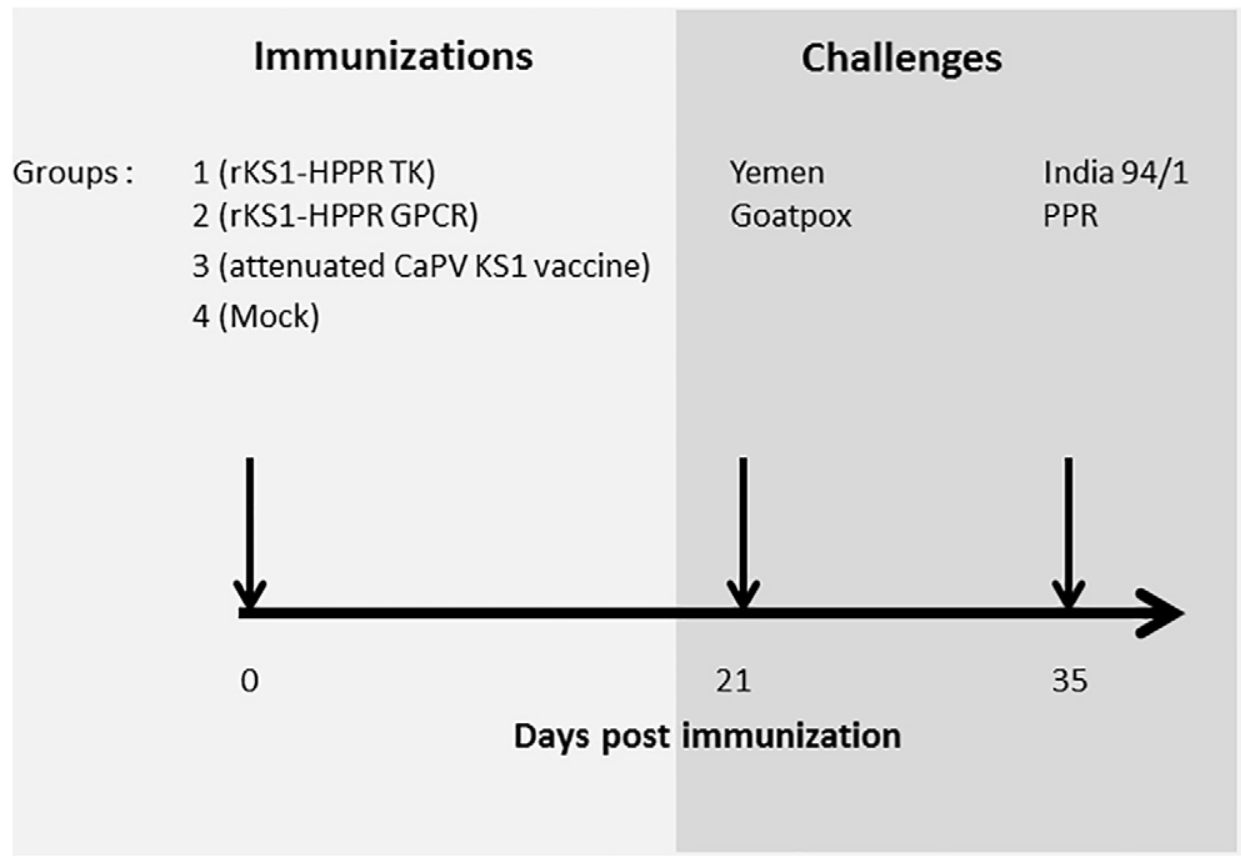

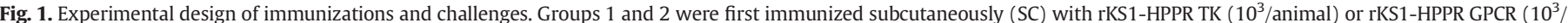

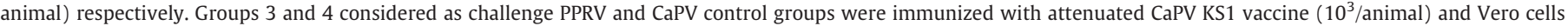

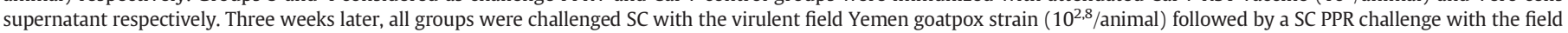
strain PPRV India 94 (10 3,8 animal) two weeks later. The timing of immunizations and challenges are indicated by arrows. 


\section{a. CaPV Clinical scores}

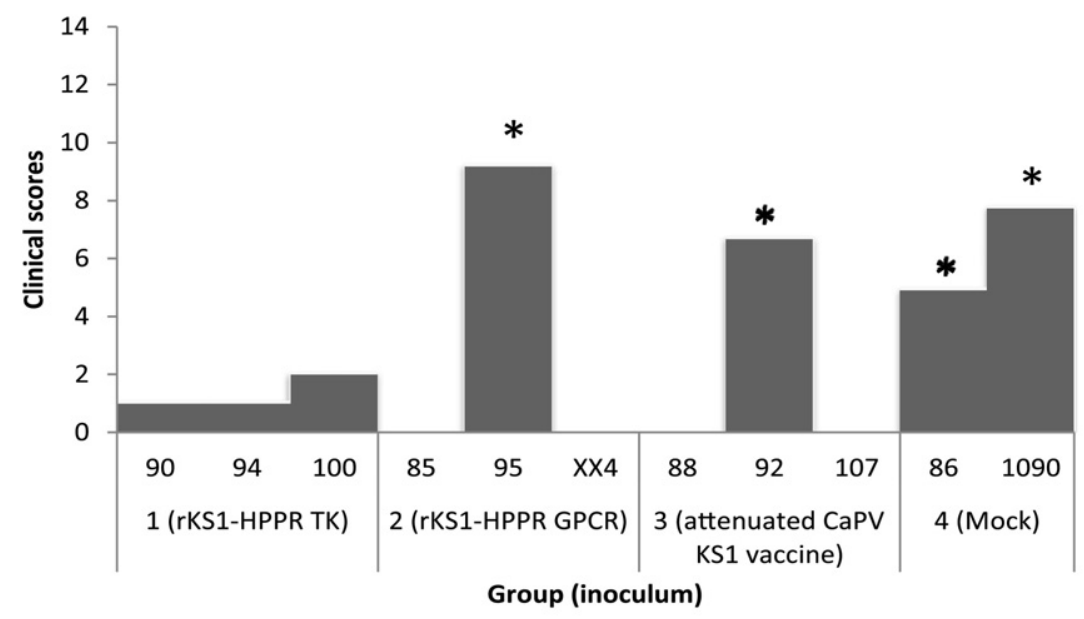

\section{b. PPR clinical scores}

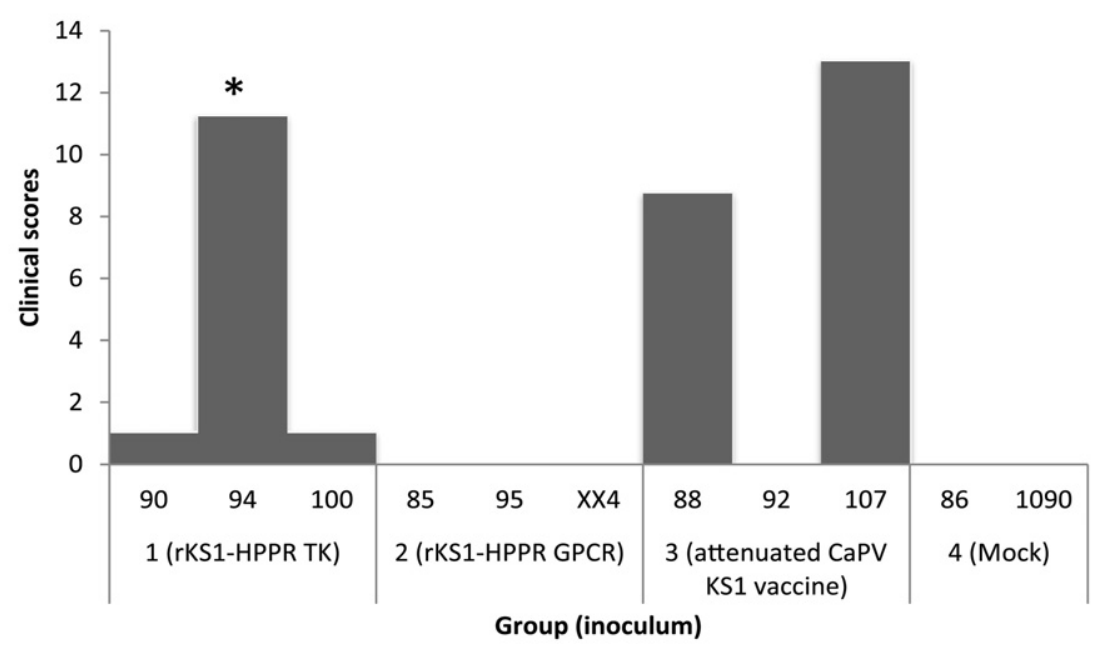

Fig. 2. Scores of clinical signs. Clinical signs including mortality were scored for each animal in the experiment. ${ }^{*}$ means death.

the CaPV KS1 vaccine group considered as the PPRV challenge control group had severe clinical signs with a score of up to 13 points (Fig. 2) and the two animals of the mock group considered as the CaPV challenge control group died.

After the CaPV challenge, one animal out of three in each of the rKS1HPPR-GPCR, attenuated CaPV KS1 vaccine groups developed severe clinical signs or died at days 10 and 11 post challenge respectively, attesting to partial protection against CaPV in the tested group (Fig. 2). The CaPV KS1 vaccine has proved for many years its protective effect in experimental conditions and in mass vaccination programs in the field (Kitching et al. 1987). Finding clinical signs in two of the CaPV vaccinated groups (including the CaPV KS1 vaccine group) while the two animals of the control group died suggest a possible overdosage of the viral challenge inoculum.

After the PPRV challenge, in the rKS1-HPPR-TK group, one animal out of three (Goat ID Number 94) died at day 12 post challenge without developing any specific anti-PPRV antibodies, whereas all the animals in the group rKS1-HPPR-GPCR developed specific anti-PPRV antibodies without any clinical signs, attesting to full protection of the animals against PPR (Table 1, Fig. 2). The unexpected death of that particular goat in the rKS1-HPPR-TK group remains unexplained.
Table 1

PPRV antibody detection in goats after recombinant CaPV rKS1-HPPR TK or rKS1-HPPR GPCR immunizations followed by CaPV and PPR challenges.

\begin{tabular}{|c|c|c|c|c|c|}
\hline \multirow{2}{*}{$\begin{array}{l}\text { Goat } \\
\text { Id }\end{array}$} & \multirow[t]{2}{*}{ Group (inoculum) } & \multicolumn{4}{|c|}{ PPRV antibody detection (ELISA/VNT titers) } \\
\hline & & Preinoculation & $4 \mathrm{dppc}$ & $7 \mathrm{dppc}$ & $14 \mathrm{dppc}$ \\
\hline 90 & 1 (rKS1-HPPT TK) & Neg/Neg & Pos $/ 60$ & Pos $/ 60$ & Pos $/ 60$ \\
\hline 94 & 1 (rKS1-HPPT TK) & $\mathrm{Neg} / \mathrm{Neg}$ & $\mathrm{Neg} / \mathrm{Neg}$ & Neg/Neg & $\mathrm{Neg} / \mathrm{Neg}$ \\
\hline 100 & 1 (rKS1-HPPT TK) & $\mathrm{Neg} / \mathrm{Neg}$ & Pos/15 & Pos/30 & Pos $/ 480$ \\
\hline 85 & 2 (rKS1-HPPR GPCR) & $\mathrm{Neg} / \mathrm{Neg}$ & Neg/15 & Pos $/ 240$ & Pos $/ 480$ \\
\hline 95 & 2 (rKS1-HPPR GPCR) & $\mathrm{Neg} / \mathrm{Neg}$ & NA & NA & NA \\
\hline $\mathrm{XX} 4$ & 2 (rKS1-HPPR GPCR) & Neg/Neg & Pos/100 & Pos $/ 240$ & Pos $/ 200$ \\
\hline 88 & $\begin{array}{l}3 \text { (attenuated CaPV KS1 } \\
\text { vaccine) }\end{array}$ & $\mathrm{Neg} / \mathrm{Neg}$ & Neg/Neg & Pos/100 & Pos/480 \\
\hline 92 & $\begin{array}{l}3 \text { (attenuated CaPV KS1 } \\
\text { vaccine) }\end{array}$ & Neg/Neg & NA & NA & NA \\
\hline 107 & $\begin{array}{l}3 \text { (attenuated CaPV KS1 } \\
\text { vaccine) }\end{array}$ & Neg/Neg & Neg/Neg & Neg/Neg & \\
\hline 86 & 4 (mock) & Neg/Neg & NA & NA & NA \\
\hline 1090 & 4 (mock) & Neg/Neg & NA & NA & NA \\
\hline
\end{tabular}

Id stands for identification; dppc, days post PPR challenge, PPRV for Peste des Petits Ruminants Virus; VNT for Virus Neutralisation Test; ELISA for Enzyme Linked ImmunoSorbent Assay); NA, not available; Neg, no antibody detected; Pos, positive antibody detection. 
These preliminary protective efficacy studies validating the GPCR as a putative insertion site resulted in highly encouraging data and paves the way to proceed from a CaPV homologous recombination strategy to a multi-pathogen delivery approach. Further experiments with larger groups of animals are needed to confirm these first experimental results. The generation of CaPV-based recombinant vaccines containing immunogenic genes of pathogens of interest which affect the same geographical zone is the next step. The development and experimental and field validation of such vaccines will offer decision makers the opportunity to better control diseases.

\section{Acknowledgements}

We wish to acknowledge A. Ngangnou and G. Libeau for their critical review and discussions. This study was funded by the French Ministry of European and Foreign Affairs (FSP project no. 2003-24 "LABOVET").

\section{References}

Berhe, G., Minet, C., Le Goff, C., Barrett, T., Ngangnou, A., Grillet, C., Libeau, G., Fleming, M. Black, D.N., Diallo, A., 2003. Development of a dual recombinant vaccine to protect small ruminants against Peste-des-Petits-ruminants virus and capripoxvirus infections. J. Virol. 77, 1571-1577.

Boshra, H., Truong, T., Nfon, C., Gerdts, V., Tikoo, S., Babiuk, L.A., Kara, P., Mather, A., Wallace, D. Babiuk, S., 2013. Capripoxvirus-vectored vaccines against livestock diseases in Africa. Antivir. Res. 98, 217-227.

Cao, J.X., Gershon, P.D., Black, D.N., 1995. Sequence analysis of HindIII Q2 fragment of capripoxvirus reveals a putative gene encoding a G-protein-coupled chemokine receptor homologue. Virology 209, 207-212.
Caufour, P., Rufael, T., Lamien, C.E., Lancelot, R., Kidane, M., Awel, D., Sertse, T., Kwiatek, O., Libeau, G., Sahle, M., Diallo, A., Albina, E., 2014. Protective efficacy of a single immunization with capripoxvirus-vectored recombinant peste des petits ruminants vaccines in presence of pre-existing immunity. Vaccine $32,3772-3779$.

Diallo, A., 2003. Control of peste des petits ruminants: classical and new generation vaccines. Dev. Biol. 114, 113-119.

Diallo, A., Minet, C., Berhe, G., Le Goff, C., Black, D.N., Fleming, M., 2002. Goat immune response to capripox vaccine expressing the hemagglutinin protein of peste des petits ruminants. Ann. N. Y. Acad. Sci. 969, 88-91.

Kitching, R.P., Hammond, J.M., Taylor, W.P., 1987. A single vaccine for the control of capripox infection in sheep and goats. Res. Vet. Sci. 42, 53-60.

Le Goff, C., Lamien, C.E., Fakhfakh, E., Chadeyras, A., Aba-Adulugba, E., Libeau, G., 2009. Capripoxvirus G-protein-coupled chemokine receptor: a host-range gene suitable for virus animal origin discrimination. J. Gen. Virol. 90, 1967-1977.

Libeau, G. Prehaud, C. Lancelot, R., Colas, F., Guerre, L, Bishop, D.H., 1995. Development of a competitive ELISA for detecting antibodies to the peste des petits ruminants virus using a recombinant nucleoprotein. Res. Vet. Sci. 58, 50-55.

OIE, 2012. Manual of Diagnostic Tests and Vaccines for Terrestrial Animals. seventh ed. World Organization for Animal Health, Paris.

Perrin, A., Albina, E., Bréard, E., Sailleau, C., Promé, S., Grillet, C., 2007. Recombinant capripoxviruses expressing proteins of bluetongue virus: evaluation of immune responses and protection in small ruminants. Vaccine 25, 6774-6783.

Rossiter, P.B., Jessett, D.M., Taylor, W.P., 1985. Microneutralisation systems for use with different strains of peste des petits ruminants virus and rinderpest virus. Trop. Anim. Health Prod. 17, 75-81.

Soi, R.K., Rurangirwa, F.R., McGuire, T.C., Rwambo, P.M., DeMartini, J.C., Crawford, T.B., 2010. Protection of sheep against Rift Valley fever virus and sheep poxvirus with a recombinant capripoxvirus vaccine. Clin. Vaccine Immunol. 17, 1842-1849.

Wallace, D.B., Viljoen, G.J., 2005. Immune responses to recombinants of the South African vaccine strain of lumpy skin disease virus generated by using thymidine kinase gene insertion. Vaccine 23, 3061-3067. 